Fábrica, G.; Bermúdez, G.; Silva-Pereyra, V.; Alonso, R. (2021) Knee Extension, the Most Important Factor for CMJ Evaluations in Volleyball Players. Revista Internacional de Medicina y Ciencias de la Actividad Física y el Deporte vol. 21 (82) pp. 211-222 Http://cdeporte.rediris.es/revista/revista82/artrodilla1242.htm

DOI: https://doi.org/10.15366/rimcafd2021.82.001

\title{
ORIGINAL
}

\section{EXTENSIÓN DE RODILLA, FACTOR MÁS IMPORTANTE DURANTE EVALUACIONES CON CMJ EN JUGADORES DE VOLEIBOL}

\section{KNEE EXTENSION, THE MOST IMPORTANT FACTOR FOR CMJ EVALUATIONS IN VOLLEYBALL PLAYERS}

\author{
Fábrica, G. ${ }^{1}$; Bermúdez, G. ${ }^{2}$; Silva-Pereyra, V. ${ }^{3}$ y Alonso, R. ${ }^{4}$ \\ 1 Dr. Ciencias Biológicas. Depto. Biofísica, Facultad de Medicina, Universidad de República \\ (Uruguay) cgfabrica@gmail.com \\ 2 Lic. Neurofisiología Clínica. Instituto Universitario Asociación Cristiana de Jóvenes Uruguay \\ (Uruguay) gbermudez.neuro@gmail.com \\ ${ }^{3} \mathrm{Mg}$. Ciencias Biológicas. Instituto Universitario Asociación Cristiana de Jóvenes, Uruguay. Depto. \\ Biofísica, Facultad de Medicina, Universidad de República (Uruguay) vlntnslv@gmail.com \\ 4 Ingeniero. Depto. Métodos Cuantitativos, Facultad de Medicina, Universidad de República \\ (Uruguay) alonso@fmed.edu.uy
}

Código UNESCO / UNESCO code: 240604 Biomecánica / Biomechanics Clasificación Consejo de Europa / Council of Europe classification: 3. Biomecánica del deporte / Biomechanics of sport

Recibido 30 de abril de 2019 Received April 30, 2019

Aceptado 1 mayo de 2020 Accepted May 1, 2020

\section{RESUMEN}

En este trabajo se evalúa cuales variables, dentro de las que resultan más entendibles para un atleta, resultan predictoras de la altura del salto con contramovimiento (CMJ). Doce jugadores de voleibol amateur $(24,5 \pm 2,7$ años; 1,85 $\pm 0,6 \mathrm{~m} ; 80,3 \pm 5,2 \mathrm{~kg}$; mean $\pm \mathrm{SD}$ ) participaron del estudio. Se evaluaron las relaciones entre 15 variables cinemáticas y la altura del salto usando el coeficiente de correlación se Spearman. Con un modelo de regresión multivariado, fue determinado que la velocidad máxima de extensión de la rodilla y el tiempo de transición durante el apoyo son predictores independientes. Se concluyó que la velocidad de extensión de rodilla es el factor más importante para la altura del salto 
y se observó que cambios dentro del rango tolerado para la acción de la rodilla, podrían afectar las evaluaciones basadas en la altura máxima del CMJ.

PALABRAS CLAVE: Saltos verticales; cinemática; rendimiento; técnica.

\begin{abstract}
This paper evaluates which variables, among those that are more understandable for an athlete, are predictors of jump height with countermovement (CMJ). Twelve amateur volleyball players $(24.5 \pm 2.7$ years; $1.85 \pm 0.6 \mathrm{~m} ; 80.3 \pm 5.2 \mathrm{~kg}$; mean \pm SD) participated in the study. The relationships between 15 kinematic variables and jump height were evaluated using Spearman's correlation coefficients. With a multivariate regression model, it was determined that the maximum knee extension velocity and the transition time are the independent predictors. It was concluded that the knee extension speed is the most important factor for the height of the jump. Changes within the range tolerated for knee action could affect the evaluations based on the maximum height of the CMJ.
\end{abstract}

KEY WORDS: Vertical jump; kinematics; performance; technique.

\title{
1. INTRODUCCIÓN
}

La altura alcanzada en un salto con contramovimiento (CMJ) es utilizada desde hace muchas décadas para evaluar capacidades en el deporte (Komi \&Bosco, 1978; Bosco, 2007; Dal Pupo et al., 2012; Petrigna et al., 2019). A partir del desplazamiento vertical del centro de masa del cuerpo (COM) durante el tiempo de contacto con el suelo se pueden definir tres fases en el CMJ. La fase de contramovimiento durante la cual el COM desciende, seguida por una fase de transición y finalmente una fase de empuje durante la cual el COM asciende (Bosco, 2007). En diferentes abordajes se muestra que las acciones motoras durante las primeras dos fases afectan la tercera (Anderson \& Pandy, 1993; Bobbert et al., 1996; Aboodara et al., 2013). Sin embargo, la altura alcanzada queda determinada por la velocidad lineal del COM en el instante del despegue (Samozino et al., 2010; Winter et al., 2016; Ruddock \& Winter 2016; Ferraro \& Fábrica, 2017), y la misma depende de la transferencia de velocidad angular de los segmentos corporales a velocidad lineal del COM durante la fase de empuje (Bobbert \&van Soest, 2001). En base a este supuesto, un análisis cinemático de variables angulares, durante la fase de empuje resulta suficiente para explicar la altura alcanzada en un CMJ.

Varios estudios han evaluado la contribución relativa de diferentes variables cinéticas y cinemáticas en la altura del CMJ (Dowling \& Vamos, 1993; AragonVargas, 1997; Ferragut et al., 2003; González-Badillo \& Marques, 2010; McErlainNaylor et al., 2014; Johnston et al., 2015; Sánchez-Sixto et al., 2019), pero no existe un consenso respecto a cuál o cuáles variables son las más importantes. Además, las variables frecuentemente utilizadas en las investigaciones, por ejemplo potencia 
y troque articular, no resultan demasiado útiles en el momento de realizar indicaciones a un atleta. En ese sentido, la duración de las fases, los rangos y las velocidades angulares de las articulaciones resultan informaciones más entendibles. Respecto a la importancia de la duración de las fases, la bibliografía muestra que no existe una respuesta clara (Aragon-Vargas, 1997; Ferragut et al., 2003; González-Badillo \& Marques, 2010). Por su parte, aunque la importancia del análisis de las acciones de las articulaciones durante el empuje aparece claramente justificado desde el punto de vista teórico en Bobbert \& van Soest, (2001). Existen diferentes opiniones respecto de la contribución de la cadera, rodilla y tobillo en la altura del CMJ. Por ejemplo, Hubley \&Wells (1983) establecieron que la rodilla es la articulación que más contribuye ( $49 \%$ del trabajo positivo total), mientras que Fukashiro y Komi (1987), remarcan la contribución de la cadera (51\%) y Vanezis \& Lees (2005) destacan la contribución de ambas (30\% rodilla y $42 \%$ cadera). Otros trabajos en cambio indican la importancia de la acción del tobillo (Mc Erlain-Naylor et al., 2014; Johnston et al., 2015).

Por otro lado, durante las evaluaciones de campo, el control de la técnica del CMJ se realiza en base a la máxima flexión de rodilla y el control de la cadera durante el empuje (Hébert-Losier et al., 2014; Sánchez-Sixto et al., 2019). Para el caso de la rodilla, existe una tolerancia de $\pm 5^{\circ}$ en la flexión máxima, aunque dicha tolerancia no está claramente justificada (Fábrica et al., 2013; Bermúdez \& Fábrica, 2014) y no suele haber un control de la situación articular en el momento del despegue. Por lo tanto, es posible que los diferentes resultados en la altura alcanzada estén influidos por pequeños cambios en la acción de las articulaciones más proximales de miembros inferiores, en lugar de reflejar diferencias en las capacidades que se propone evaluar con la altura del CMJ. Moran\& Wallace (2007), encontraron que al aumentar $20^{\circ}$ el rango de rodilla la altura del CMJ mejora un $17 \%$. Sin embargo, aún es necesario discutir con mayor profundidad el efecto que podrían tener las pequeñas variaciones angulares sobre los resultados de las evaluaciones. Identificar variables predictoras de la altura del $\mathrm{CMJ}$ dentro de aquellas que permiten realizar indicaciones a un atleta y conocer si las variaciones angulares dentro del rango de tolerancia considerado habitualmente pueden llevar a diferentes interpretaciones durante las evaluaciones, es información relevante tanto para atletas como entrenadores.

\section{OBJETIVOS}

Este estudio tuvo como primer objetivo identificar las acciones de las extremidades inferiores que predicen la altura alcanzada en un CMJ dentro de un conjunto de variables que los atletas pueden reconocer e interpretar fácilmente. En segunda instancia determinar si el resultado de la evaluación puede verse afectado cuando no se realiza un control cuantitativo de la técnica del salto. 


\section{MÉTODOS}

Doce jugadores de voleibol amateur $(24,5 \pm 2,7$ años; $1,85 \pm 0,6 \mathrm{~m} ; 80,3 \pm 5,2 \mathrm{~kg}$; mean \pm SD) con más de 5 años de experiencia en el deporte y una frecuencia de entrenamiento de 3 veces por semana, participaron en este estudio. El tamaño de la muestra para este trabajo se estimó con base en los datos reportados en estudios previos (Markovic\&Jaric, 2007; Pazin et al., 2013), y en las guías de Cohen (1988), con un nivel alfa de 0.05 y un nivel de potencia de 0.8 . Todos los sujetos contaban con experiencia en evaluaciones con $\mathrm{CMJ}$, no sufrieron lesiones durante los 6 meses previos al estudio, ni fueron sometidos alguna vez a cirugía en las extremidades inferiores. Los participantes fueron informados sobre los objetivos y las características del estudio, sus consentimientos fueron previamente obtenidos. El estudio fue realizado siguiendo los requerimientos estipulados en la Declaración de Helsinki 2013, el protocolo y el consentimiento informado fue aprobado por el comité de ética local (número de aprobación 07114000176409).

Los datos fueron registrados en un mismo día durante el periodo precompetitivo. Durante los registros los voluntarios usaron su calzado habitual de entrenamiento. Luego de 10 minutos de calentamiento autoseleccionado y tres saltos submáximos, los participantes realizaron cinco CMJ máximos. Se les indicó realizar los saltos del mismo modo que en las evaluaciones de campo. Los individuos comenzaron de una posición erguida y realizaron el contramovimiento, hasta alcanzar un ángulo de flexión de rodilla próximo a $90^{\circ}$, con las manos sobre la cintura durante todo el movimiento. Posteriormente, los tres mejores saltos (máxima altura alcanzada) de cada sujeto fueron seleccionados para los análisis. Cuarenta y nueve marcadores reflexivos fueron posicionados en puntos anatómicos de referencia en cada participante. Este número de marcadores permite tener una reconstrucción precisa del COM (Fábrica et al., 2019) y por tanto mayor precisión en la determinación la altura del salto, que fue la variable dependiente en este estudio. Ocho cámaras Bonita (1 MPx cámara óptica, $250 \mathrm{fps}$ con lente varifocal $(4$ a 12mm) y NIR estroboscópico) conectadas a VICON MOTION SYSTEMS (Nexus 2.5) (Oxford Metrics $L t d$ ) fueron usadas para obtener la reconstrucción 3-d del movimiento. La trayectoria de los marcadores fue suavizada usando un filtro Butterworth de cuarto orden, frecuencia de corte $6 \mathrm{~Hz}$ sin retardo, y fueron exportadas a MATLAB R2017a ${ }^{\circledR}($ Mathworks, Inc.) para determinar las variables.

Se seleccionaron quince variables cinemáticas (variables independientes) para analizar su asociación con la altura alcanzada en un CMJ (variable dependiente). Antes de usar los valores promedios de ambos miembros, se verificó si se podía asumir simetría bilateral. Para esto se calculó el índice de simetría (LSI) para cada variable como: valor de la extremidad izquierda /valor de la extremidad derecha $\mathrm{x}$ 100. El déficit de simetría fue definido por LSI< $90 \%$. Los ángulos fueron considerados negativos para la flexión plantar del tobillo y para la extensión de rodilla y cadera. 
Rev.int.med.cienc.act.fís.deporte - vol. 21 - número 82 - ISSN: 1577-0354

Tabla 1. Definición de las variables analizadas y sus abreviaciones correspondientes.

Abreviación Definición

de la variable

\begin{tabular}{ll}
$\mathrm{HJ}$ & Altura máxima alcanzada en el salto, determinada a partir de la diferencia \\
& del máximo del componente vertical de la posición del COM y el valor de \\
& dicho componente en el instante de despegue. \\
$\mathrm{Tc}$ & Duración de la fase de contramovimiento \\
$\mathrm{Tt}$ & Duración de la fase de transición \\
$\mathrm{Tp}$ & Duración de la fase de empuje \\
$\mathrm{Hr}$ & Rango angular promedio de la cadera en el plano sagital durante el \\
& empuje. \\
$\mathrm{Kr}$ & Rango angular promedio de la rodilla en el plano sagital durante la fase de \\
& empuje. \\
$\mathrm{Ar}$ & Rango angular promedio del tobillo en el plano sagital durante el empuje. \\
$\mathrm{VHm}$ & Velocidad angular media de la cadera en el plano sagital durante el \\
$\mathrm{VKm}$ & empuje. \\
$\mathrm{VAm}$ & Velocidad angular media de la rodilla en el plano sagital durante el \\
$\mathrm{VHp}$ & empuje. \\
$\mathrm{VKp}$ & Velocidad angular media del tobillo en el plano sagital durante el empuje. \\
$\mathrm{VAp}$ & Velocidad angular máxima de cadera en el plano sagital durante el \\
$\mathrm{Htp}$ & Vempuje. \\
$\mathrm{Ktp}$ & Velocidad angular máxima de rodilla en el plano sagital durante el empuje. \\
$\mathrm{Atp}$ & Tiempo de la velocidad angular máxima de cadera durante el empuje. \\
& Tiempo de la velocidad angular máxima de rodilla durante el empuje. \\
& Tiempo de la velocidad angular máxima de tobillo durante el empuje. \\
\hline
\end{tabular}

\subsection{ANÁLISIS ESTADÍSTICO}

Las medias y los desvíos estándar (SD) fueron calculados para todas las variables. La intensidad de la asociación entre cada variable cinemática y la altura del salto se determinaron con el coeficiente de correlación de Spearman. Se evaluó un modelo lineal multivariado para predecir la $\mathrm{HJ}$. Las variables con $\mathrm{p}<0,10$ en el modelo univariado fueron incluidas en el modelo multivariado. Se utilizó stepwise backwardselection con nivel de significación de remoción de 0,1 , se evaluó la muticolinealidad con el coeficiente de inflación de varianza (VIF). Luego de definir el modelo final, se calculó la correlación parcial para cada una de las variables predictoras. Las pruebas Breusch-Pagan/Cook-Weisberg fueron realizadas para evaluar la heterocedasticidad y Shapiro-Wilks para la normalidad de los datos. El nivel de significancia estadística usado fue $\alpha=0,05$. El análisis de datos fue realizado en los programas Stata 13 y $R$.

\section{RESULTADOS}

Para todas las variables analizadas no hubo déficit de simetría, por lo tanto, se utilizó el valor medio para ambas extremidades en todas las variables relacionadas con los ángulos articulares. 
Los valores obtenidos para las quince variables independientes consideradas en este estudio y $\mathrm{HJ}$ se presentan en la tabla 2, conjuntamente con los correspondientes coeficientes de correlación obtenidos entra cada variable y $\mathrm{HJ}$. Las correlaciones reflejan que la $\mathrm{HJ}$ estuvo correlacionada con; $\mathrm{Tt}, \mathrm{Hr}, \mathrm{Kr}, \mathrm{VKm}$, VKp y VHm.

Los resultados de los análisis univariados y multivariados se presentan en la tabla 3. La ecuación obtenida con el modelo del análisis multivariado es:

$$
H J=0,0129+0,0006(-V K p)-1,6853 \mathrm{Tt}, \quad\left(R^{2}=0,65\right) .
$$

La figura 1 muestra la relación entre $\mathrm{HJ}$ y las dos variables predictoras del modelo final. La correlación parcial entre HJ y VKp es de $-0,73$ y entre $\mathrm{HJ}$ y Tt de $-0,31$. La correlación negativa entre HJ y VKp se debe al criterio asumido para la extensión angular.

Tabla 2. Análisis descriptivo de las variables y coeficiente de correlación de Spearman rs ( $n=36)$.

\begin{tabular}{cccc}
\hline & Mean & SD & Correlation \\
\hline $\mathrm{HJ}(\mathrm{m})$ & 0,383 & 0,041 & \\
$\mathrm{Tc}(\mathrm{s})$ & 0,460 & 0,043 & $-0,22$ \\
$\mathrm{Tt}(\mathrm{s})$ & 0,032 & 0,005 & $-0,51^{*}$ \\
$\mathrm{Tp}(\mathrm{s})$ & 0,266 & 0,022 & 0,07 \\
$\operatorname{VHp}\left({ }^{\circ} / \mathrm{s}\right)$ & $-509,288$ & 34,070 & $-0,19$ \\
$\operatorname{VKp}\left({ }^{\circ} / \mathrm{s}\right)$ & $-723,648$ & 48,344 & $-0,78^{*}$ \\
$\operatorname{VAp}\left({ }^{\circ} / \mathrm{s}\right)$ & $-597,997$ & 149,119 & $-0,14$ \\
$\mathrm{Htp}(\mathrm{s})$ & 0,247 & 0,025 & 0,01 \\
$\mathrm{Ktp}(\mathrm{s})$ & 0,259 & 0,024 & 0,04 \\
$\operatorname{Atp}(\mathrm{s})$ & 0,26 & 0,025 & 0,04 \\
$\operatorname{Hr}\left({ }^{\circ}\right)$ & $-67,713$ & 7,103 & $-0,58^{*}$ \\
$\operatorname{Kr}\left({ }^{\circ}\right)$ & $-72,713$ & 9,597 & $-0,49^{*}$ \\
$\operatorname{Ar}\left(^{\circ}\right)$ & $-44,946$ & 7,591 & $-0,09$ \\
$\operatorname{VHm}\left({ }^{\circ} / \mathrm{s}\right)$ & $-254,853$ & 24,170 & $-0,55^{*}$ \\
$\operatorname{VKm}\left({ }^{\circ} / \mathrm{s}\right)$ & $-273,792$ & 35,393 & $-0,42^{*}$ \\
$\operatorname{VAm}\left({ }^{\circ} / \mathrm{s}\right)$ & $-170,317$ & 34,861 & $-0,05$ \\
\hline${ }^{*}=\mathrm{p}<0,05$ & & &
\end{tabular}

Tabla 3. Resultado del modelo de regresión lineal.(coeficientes no estandarizados)

\begin{tabular}{|c|c|c|c|c|c|c|}
\hline Variable & Univariado & Std. Err & $\mathrm{p}$-valor & Multivariado* & Std. Err & $\mathrm{p}$-valor \\
\hline $\mathrm{Tt}(\mathrm{s})$ & $-3,9964$ & 1,15058 & 0,001 & $-1,6853$ & 0,87890 & 0,064 \\
\hline VKp ( $\%$ ss) & $-0,0006$ & 0,00008 & 0,001 & $-0,0006$ & 0,00009 & 0,001 \\
\hline $\operatorname{Hr}\left({ }^{\circ}\right)$ & $-0,0030$ & 0,00079 & 0,001 & & & \\
\hline $\operatorname{Kr}\left({ }^{\circ}\right)$ & $-0,0021$ & 0,00063 & 0,001 & & & \\
\hline $\mathrm{VHm}(\% / \mathrm{s})$ & $-0,0009$ & 0,00024 & 0,001 & & & \\
\hline VKm ( $\%$ s) & $-0,0005$ & 0,00017 & 0,001 & & & \\
\hline
\end{tabular}



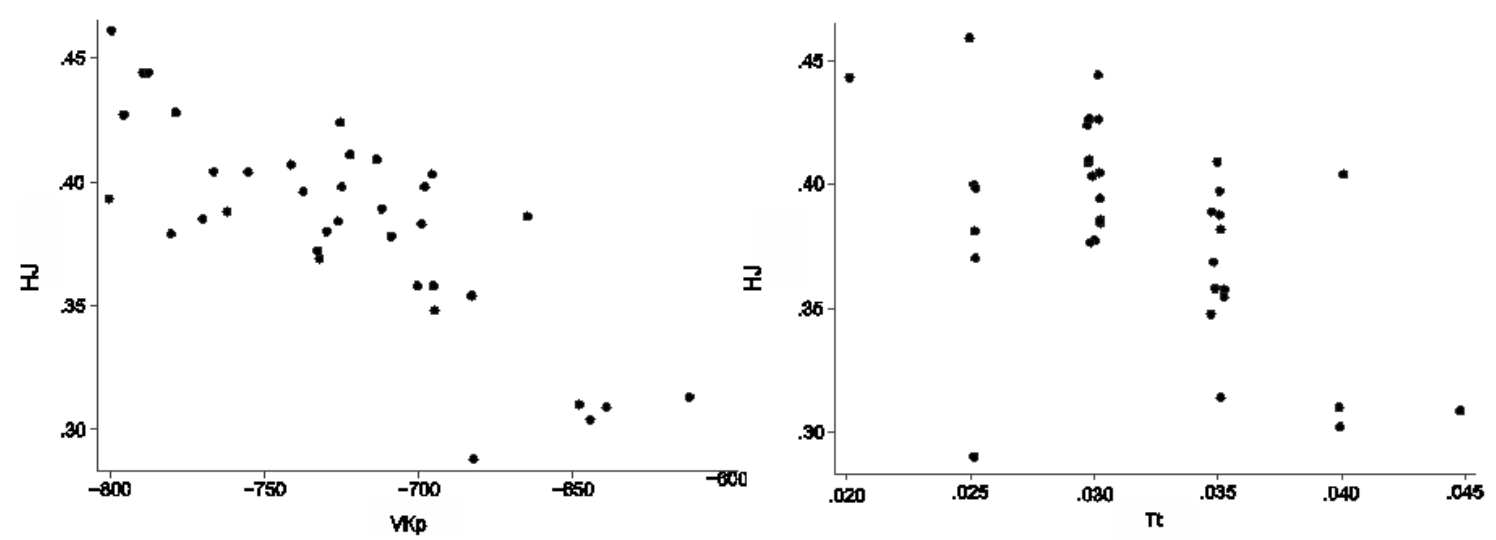

Figura 1. Relación entre la altura del salto con contramovimiento y las dos variables independientes predictoras en este estudio: máxima velocidad de extensión de rodilla (VKp) y duración de la fase de transición (Tt). Como Tt es indicada una variable importante en artículos previos, apoyándonos en el hecho que su significación fue próxima al $5 \%$ y una leve mejora en el ajuste se optó por dejarla en el modelo. El R2 ajustado incluyendo Vkp es de 0,60, al incluir Tt pasa a 0,63 .

\section{DISCUSIÓN}

En este estudio se identificaron variables predictoras de la altura del $\mathrm{CMJ}$, dentro de aquellas variables cinemáticas que los atletas pueden interpretar. Además, se analizó si cambios en la ejecución de la técnica dentro del rango habitualmente tolerado, podrían afectar las evaluaciones basadas en la altura del salto. Aunque todas las variables consideradas en este estudio han sido previamente analizadas en el CMJ, el foco específico, así como las principales preguntas abordadas no se han considerado en estudios anteriores.

La HJ encontrada en este estudio fue menor a la reportada en trabajos previos (González-Badillo\& Marques, 2010; McErlain-Naylor et al., 2014; Sánchez-Sixto et al., 2019). Esa menor altura puede estar asociada con las características de nuestra muestra, atletas amateur que entrenan tres veces por semana. De hecho, los valores en de $\mathrm{HJ}$ se acercan bastante a los reportados en trabajos donde se analizan sujetos físicamente activos no deportistas (Jurado-Lavanant et al., 2017).

En cuanto a las asociaciones encontradas entre la altura y las variables independientes consideradas un primer punto a destacar es que dentro de las variables temporales solamente Tt correlacionó con $\mathrm{HJ}$ de manera estadísticamente significativa. La duración de las fases de contramovimiento y empuje no presentaron asociaciones estadísticamente significativas con la altura. Esos resultados, se contraponen a otros trabajos donde se ha señalado la importancia del contramovimiento (Ferragut et al., 2003) y de la fase de empuje (Dowling \& Vamos, 1993; Bermúdez \& Fábrica, 2014) en la altura del salto. En ese sentido nuestros resultados se acercan más a lo establecido por González-Badillo y Marques (2010), quienes discutieron que la duración de las fases excéntrica y concéntrica son 
indicadores débiles de la performance del CMJ. El aspecto más importante a considerar respecto a la duración de las fases es que entre Tt y HJ existe una correlación negativa. Esto se puede interpretar como que una disminución del tiempo de transición permite un mejor aprovechamiento del ciclo acortamientoestiramiento, aunque en realidad la correlación parcial entre HJ y Tt fue baja.

Para identificar las variables independientes predictivas de la $\mathrm{HJ}$ se implementó un modelo de regresión multivariado. Las variables Tt y VKp juntas explican el $65 \%$ de la altura alcanzada. Dentro de las variables angulares que fueron analizadas, (VKp) fue la única que permaneció en el modelo final con $\mathrm{HJ}$. El modelo indica que a mayor velocidad de extensión de rodilla, mayor es la altura del salto. Ese resultado es acorde con lo reportado por Hubley y Wells (1983), quienes indicaron que la rodilla contribuye en un alto porcentaje $(49 \%)$ al trabajo mecánico realizado durante el CMJ.Este resultado también es acorde con estudios que indican que la potencia de rodilla es decisiva en la altura de los saltos verticales (Aragon-Vargas, 1997; McErlain-Naylor et al., 2014; Johnston et al., 2015). Aunque nosotros no medimos potencia articular, los altos valores de velocidad máxima angular pueden ser asociados con los altos valores de potencia (Young et al., 1999). Sin embargo, los trabajos de McErlain-Naylor et al., (2014) y Johnston et al., (2015) destacan también la importancia de la acción del tobillo como predictor de la altura del CMJ. Que no se detectara la influencia de la acción del tobillo en HJ constituye una de las diferencias más destacadas de nuestros resultados con respecto a lo encontrado en estudios previos. Aquí nuevamente debemos considerar que las características de la muestra estudiada pueden estar influyendo.

Aunque es esperable que atletas amateur y profesionales usen técnicas de saltos semejantes (Vanezis \& Lees, 2005; McErlain-Naylor et al., 2014), existe la posibilidad de que en atletas profesionales, el último segmento de la cadena cinemática contribuya en mayor medida a la altura del CMJ que en deportistas amateur como los que hemos estudiado.Esta diferencia puede deberse también a que los estudios de referencia (McErlain-Naylor et al., 2014; Johnston et al., 2015) utilizan variantes en la técnica de CMJ respecto a los saltos utilizados en nuestro estudio. Esos cambios de técnica, por ejemplo, en posición de las manos, pueden ser la explicación de muchas de las diferencias encontradas (Blache \& Monteil, 2013; Petrigna et al., 2019).

Un aspecto importante que surge de este estudio es que un control no riguroso de la rodilla puede afectar el resultado de la evaluación. Aunque la indicación fue alcanzar una máxima flexión de rodilla de $90^{\circ}, \mathrm{Kr}$ estuvo por debajo de ese valor. El valor de $\mathrm{Kr}$ se puede alterar tanto por no alcanzar la flexión de $90^{\circ}$ como por una mayor flexión de rodilla al momento del despegue. En un estudio reciente SánchezSixto et al. (2019), discuten el efecto del desplazamiento del COM en las diferencias en la altura de saltos verticales y sugieren la necesidad de examinar el desplazamiento del COM para interpretar adecuadamente las diferencias entre el SJ y el CMJ cuando el criterio establecido es $90^{\circ}$ de flexión de rodilla. 
Por su parte, Moran \& Wallace (2007), encontraron que aumentar el rango de movimiento de la rodilla de $70^{\circ}$ a $90^{\circ}$ resulta en un aumento del $17 \%$ de la altura del salto. Dado que nuestros valores estuvieron dentro de ese rango, podríamos suponer que, si los sujetos hubieran saltado más cerca del rango esperado, la altura seria próxima a la reportada en trabajos previos realizados con atletas (GonzálezBadillo \& Marques, 2010; McErlain-Naylor et al., 2014). Por otra parte, el principal predictor de la altura del salto encontrado en este trabajo (VKp), presenta una fuerte correlación con $\mathrm{Kr}$ y $\mathrm{Hr}$. En base a esto podemos decir que cambios en el rango de movimiento de estas dos articulaciones podría estar influenciando VKp, variando así la altura del salto y por tanto la interpretación que surge de este parámetro. En las evaluaciones con CMJ, los atletas son instruidos a restringir el movimiento de la cadera y alcanzar $90^{\circ}$ en la articulación de rodilla. Estas restricciones tienen el propósito de utilizar siempre la misma técnica. Nuestros resultados indican que el control de la técnica debería incluir una cuantificación del rango angular de las articulaciones más proximales.

En resumen, nuestros resultados nos llevan a la idea de que para lograr una buena altura en el CMJ los atletas se deben enfocar principalmente en dos cosas, lograr una rápida fase de transición, lo cual ya ha sido discutido en trabajos previos, y extender las rodillas lo más rápido posible durante el empuje, siendo este último el factor más importante. Este es un resultado con posible interés práctico en el sentido que sugiere que la coordinación puede estar influyendo significativamente en la evaluación de las capacidades físicas mediante la altura del CMJ. Además, los resultados de nuestro estudio sugieren que el control de la técnica del CMJ debe ser más estricto e incluir cuantificaciones del rango angular, de no hacerlo se puede llegar a una interpretación equivocada de las capacidades evaluadas con el CMJ. Creemos que esto cuestiona, o al menos advierte sobre la real utilidad de muchos sistemas de evaluación que no permiten realizar un control cuantitativo de los valores angulares durante el salto. Es posible que la evaluación de la fuerza manifestada durante el CMJ dependa de esas sutiles diferencias en la técnica y dada la enorme cantidad de asociaciones establecidas entre la altura alcanzada en un CMJ y diferentes capacidades, se debería analizar con mayor profundidad este tema. Sería interesante entre otras cosas extender este tipo de estudio a otras poblaciones y buscar herramientas que expliquen mejor la relación entre coordinación articular y altura del salto.

\section{CONCLUSIONES}

La altura alcanzada en un CMJ en jugadores de voleibol amateur depende principalmente de la velocidad con la cual la rodilla es extendida durante el tiempo de contacto con el piso.

La utilización de la altura del CMJ como una herramienta de evaluación sin un control cuantitativo de la técnica debería ser reconsiderada. 


\section{REFERENCIAS BIBLIOGRAFICAS}

Aboodara SJ, Yusof A, Osman NAA, Thompson MW, Mokhtar AH. Enhanced performance with elastic resistance during the eccentric phase of a countermovement jump. Int. J Sports Physiol Perform. 2013; 8:181-187. https://doi.org/10.1123/ijspp.8.2.181

Anderson FC, Pandy MG. Storage and utilization of elastic strain energy during jumping. J Biomech 1993; 26:1413-1427. https://doi.org/10.1016/00219290(93)90092-S

Aragon-Vargas L, Gross MM. Kinesiological factors in vertical jump performance: Differences among individuals. J Appl Biomech. 1997;13:24-44. https://doi.org/10.1123/jab.13.1.24

Bermúdez G, Fábrica CG. Factores determinantes del rendimiento cuando el countermovement jump se realiza en fatiga aguda. Rev Bras Cineantropom Desempenho Hum. 2014;16:316-324. https://doi.org/10.5007/1980$\underline{0037.2014 v 16 n 3 p 316}$

Blache Y, Monteil K. Effect of arm swing on effective energy during vertical jumping: Experimental and simulation study. Scand J Med Sci Sports. 2013;23:121129. https://doi.org/10.1111/sms.12042

Bobber MF, Gerritsen KG, Litjens MC, Van Soest AJ. Why is countermovement jump height greater than squat jump height? Med Sci Sports Exerc. 1996;28:14021412. https://doi.org/10.1097/00005768-199611000-00009

Bobbert MF, van Soest AJ. Why do people jump the way they do? Exerc Sport Sci Rev. 2001;29:95-102 https://doi.org/10.1097/00003677-200107000-00002

Bosco, C. Forca Muscular. Sao Paulo: Phorte, 2007.

Cohen J. Statistical power analysis for the behavioral sciences. 2nd ed. New York: Lawrence Erlbaum Associates, 1988.

Dal Pupo J, Detanico D, Dos Santos S. Parâmetros cinéticos determinantes do desempenho nos saltos verticais. Rev Bras Cieneantropom e Desempenho Hum. 2012; 14:41-51. https://doi.org/10.5007/1980-0037.2012v14n6p738

Dowling JJ, Vamos L. Identification of kinetic and temporal factors related to vertical jump performance. J Appl Biomech. 1993; 9:95-110. https://doi.org/10.1123/jab.9.2.95

Fábrica CG, González P, Fagundes Loss J. Estudy of acute fatigue effects on lower limbs during countermovement jump based on force records. Motriz (UNESP). $\quad 2013 ; \quad 19: 737-745 . \quad$ https://doi.org/10.1590/S198065742013000400011

Fábrica, CG, Jerez-Mayorga, D, Silva Pereyra, V. Pendular energy transduction in the different phases of gait cycle in post-stroke subjects. Human Movement Science. 2019; 66: 521 - 528. https://doi.org/10.1016/j.humov.2019.06.006

Ferragut C, Cortadellas J, Arteaga R, Calbet JAL. Predicción de la altura de salto vertical, importancia del impulso mecánico de la masa muscular de las extremidades inferiores. Eur J Hum Mov. 2003; 10:7-22.

Ferraro D, Fábrica CG. Differences in the utilisation of active power in squat and countermovement jumps. Eur J Sport Sci. 2017; 17:673 - 680. https://doi.org/10.1080/17461391.2017.1305453 
Fukashiro S, Komi PV. Joint moment and mechanical power flow of the lower limb during vertical jump. Int $J$ Sports Med. 1987; 8:15-21. https://doi.org/10.1055/s-2008-1025699

González-Badillo JJ, Marques MC. Relationship between kinematic factors and countermovement jump height in trained track and field athletes. J Strength Cond Res. 2010; 24:3443-3447. https://doi.org/10.1519/JSC.0b013e3181bac37d

Hébert-Losier K, Jensen K, Holmberg HC. Jumping and hopping in elite and amateur orienteering athletes and correlations to sprinting and running. International Journal of Sports Physiology and Performance. 2014; 9: 993-999. https://doi.org/10.1123/ijspp.2013-0486

Hubley CL, Wells RP. A work-energy approach to determine individual joint contributions to vertical jump performance. Eur J Appl Physiol Occup Physiol. 1983; 50:247-254. https://doi.org/10.1007/BF00422163

Johnston LA, Butler RJ, Sparling TL, Queen RM. A single set of biomechanical variables cannot predict jump performance across various jumping tasks. J Strength Cond Res. 2015; 29:396-407. https://doi.org/10.1519/JSC.0000000000000779

Jurado-Lavanant A., Fernández-García JC., Pareja-Blanco F, Alvero-Cruz JR Efectos del entrenamiento pliométrico acuático vs. Seco sobre el salto vertical. Revista Internacional de Medicina y Ciencias de la Actividad Física y el Deporte. 2017; 17: 73-84.

Komi PV, Bosco C. Utilization of stored elastic energy in leg extensor muscles by men and women. Med Sci Soprts Exerc. 1978; 10:261-265.

Markovic G, Jaric S. Positive and Negative loading and mechanical output in maximum vertical jumping. Med Sci Sports Exerc. 2007; 39: 1757-1764. https://doi.org/10.1249/mss.0b013e31811ece35

McErlain-Naylor S, King M, Pain MTG. Determinants of countermovement jump performance: a kinetic and kinematic analysis. J Sports Sci.2014; 32: 18051812. https://doi.org/10.1080/02640414.2014.924055

Moran K, Wallace ES. Eccentric loading and range of knee joint motion effects on performance enhancement in vertical jumping. Hum Mov Sci. 2007; 26:824840. https://doi.org/10.1016/i.humov.2007.05.001

Pazin N, Berjan B, Nedeljkovic A, Markovic G, Jaric, S. Power output in vertical jumps: does optimum loading depend on activity profiles? Eur J Appl Physiol. 2013; 113: 577-589. https://doi.org/10.1007/s00421-012-2464-z

Petrigna L, Karsten B, Marcolin G, Paoli A, D'Antona G, Palma A, Bianco A. A Review of Countermovement and Squat Jump Testing Methods in the Context of Public Health Examination in Adolescence: Reliability and Feasibility of Current Testing Procedures.Frontiers in Physiology. 2019. https://www.frontiersin.org/article/10.3389/fphys.2019.01384 https://doi.org/10.3389/fphys.2019.01384

Ruddock AD, Winter EM. Jumping depends on impulse not power. J Sports Sci. 2016; 34:584-585. https://doi.org/10.1080/02640414.2015.1064157

Samozino P, Morin JB, Hintzy F, Belli A. Jumping ability: A theoretical integrative approach. Journal of Theoretical Biology 2010; 264:11-18. https://doi.org/10.1016/j.jtbi.2010.01.021 
Sánchez-Sixto A., Harrison AJ, Floría P. La importancia de la profundidad del contramovimiento en el ciclo estiramiento-acortamiento. Revista Internacional de Medicina y Ciencias de la Actividad Física y el Deporte. 2019; 19: 33-44. https://doi.org/10.15366/rimcafd2019.73.003

Vanezis A, Lees A. A biomechanical analysis of good and poor performers of the vertical jump. Ergonomics. 2005; 48:1594-1603. https://doi.org/10.1080/00140130500101262

Winter EM, Abt G, BrookesFBC, ChallisJH, FowlerNE, KnudsonDV, et al. Misuse of "Power" and Other Mechanical Terms in Sport and Exercise Science Research. J Strength Cond Res. 2016; 30: 292-300. https://doi.org/10.1519/JSC.0000000000001101

Young W, Wilson G, Byrne C. Relationship between strength qualities and performance in standing and run-up vertical jumps. J Sports Med Phys Fitness. 1999; 39:285-293.

Número de citas totales / Total references: 32

Número de citas propias de la revista / Journal's own references: 2

Rev.int.med.cienc.act.fís.deporte - vol. 21 - número 82 - ISSN: 1577-0354 